\title{
Referee Acknowledgement for 2014
}

In this issue, we publish the names of those who reviewed manuscripts for us in 2014.

The Editor-in-Chief, Specialist Editors and everyone involved in publishing BJC would like to extend our sincere thanks to them for contributing their expertise and time. Our referees play an invaluable role ensuring that BJC continues to publish the high quality original papers and reviews that make it one of the world's leading oncology journals.

Lauri A Aaltonen

Ossama Abbas

Ata Abbasi

Manar Elsheikh Abdel-Rahman

Mohamed Abdel-rahman

Ghassan K Abou-Alfa

Giovanni Actis

Anya Adair

David Adams

Peter Adams

Richard Adams

Lucile Adams-Campbell

Douglas James Alexander Adamson

Olivier Adotevi

Pooja Advani

JGJV Aerts

Farrukh Afaq

BB Aggarwal

Jackeline Agorreta

Marco Agostini

Joseph K Agyin

Roger Ahern

Tim A Ahles

Amrita Ahluwalia

Hashim Uddin Ahmed

Nuzhat Ahmed

Yasar Albushra Abdul Rahiem Ahmed

Sam Hjelmeland Ahmedzai

Erin Ahn

Sang Hoon Ahn

Nita Ahuja

Nicolas Aide

Jaffer A Ajani

Agneta Akesson

Suminori Akiba

Salah-Eddin Al-Batran

Fares Al-Ejeh

Michael C Alavanja

Abass Alavi

Rita CR Albuquerque

Krasimira Aleksandrova

Ramon Alemany

Dominik D Alexander

Leo Alexandre

Roberta R Alfieri

Raghib Ali

Malcolm R Alison
Martin Almquist

Amelia Altavilla

MC Alves-Guerra

Anton Amann

Ravi Amaravadi

Hesham M Amin

Zhiqiang An

Shrikant Anant

Jesper B Andersen

$\mathrm{V}$ Andersen

Annie Anderson

Roland Andersson

Fabrice Andre

Nicoll Andreescu

$\mathrm{H}$ Jervoise N Andreyev

Vasilis Androutsopoulos

Ritu Aneja

Angelica Angeles-Llerenas

Roberto Angioli

Lieven Annemans

Thunyarat Anothaisintawee

Masaki Anraku

Toni M Antalis

Alessandro Antonelli

Ana Aparicio

Andrew E Aplin

Rami I Aqeilan

Hwyda A Arafat

Marc Arbyn

Luca Arcaini

Peter A Argenta

Hendrik-Tobias Arkenau

Alexander Arlt

Andrew J Armstrong

Lisen Arnheim-Dahlstrom

Edurne Arriola

Maria Libera Ascierto

Melissa Assel

Eric Asselin

Igor Astsaturov

Gerhardt Attard

Edward F Attiyeh

Havard Attramadal

Riccardo A Audisio

K Augoff

Monique Aumailley

Philippe Autier
Kelly A Avery-Kiejda

Eli Avisar

Abdel Kareem Azab

Atiqullah Aziz

Hideo Baba

Leon A Bach

Jamie Baddum

Raffaele Baffa

Zsuzsanna Bago-Horvath

Chunxue Bai

Robert Baiocchi

Duncan Baird

Paul Baird

Laurence Baker

Carmen Balana

R Balasubramanian

Gustavo Baldassarre

David R Baldwin

Stuart Ballantyne

Cynthia Bamdad

Udai Banerji

Ulrich Christian Bang

Yung-Jue Bang

D Bansal

Silvia ML Barabino

Ludovic Barault

Andrew P Barbour

Stephen Barclay

Dalsu Baris

Christopher A Barker

Thomas Ian Barron

Rupert Bartsch

Holger Bastians

Devraj Basu

Andrew Bathgate

Elena V Batrakova

Georg Bauer

Martina Bazzaro

Ian LP Beales

Nicole Beauchemin

Michel Beaugrand

Karl-Friedrich Becker

Therese M Becker

Tomasz M Beer

Rudd LM Bekkers

Diana Bell

Claudio Belluco
Mattias Belting

Par-Ola Bendahl

Charles L Bennett

Al B Benson

Marianne Berg

Martin R Berger

Walter Berger

Thierry Berghmans

TK Bergmann

David M Berman

Ora Bernard

Mark Bernstein

Franco Berrino

Narelle Berry

Anders Bertelsen

Gaetano Bertino

Joseph R Bertino

Francesco Bertolini

Giulia Bertolini

Andrea Bertotti

Simone Bertz

Eric M Bessell

Chetan Bettegowda

Shailender Bhatia

Fabrizio Bianchi

Giampaolo Bianchini

Tina Bianco-Miotto

Frederic Bibeau

Siamak Bidel

Giuseppe Bifulco

Maurizio Bifulco

Gilbert Bigras

Ioannis Biliatis

Steven D Billings

Philippe Birembaut

Helgi Birgisson

Anupam Bishayee

Peter C Black

Roman A Blaheta

Jaishri O’Neill Blakeley

Phillip Blanchette

Giovanni Blandino

William S Blaner

Roger G Blanks

Jean-Yves Blay

Jane M Blazeby

Maaike CG Bleeker 
Harry Bleiberg

Elisabeth Bloemena

Lisa Boardman

Carla Boccaccio

Guido Bocci

Thomas Bock

Barrie Bode

Stefan Boeck

Katja Boehm

Malte Boehm

Paolo Boffetta

Ugo Boggi

Mark S Bolding

Laura Bonanno

Fiona Bonar

Melissa L Bondy

Dominique Bonnet

Petri Bono

Stefanos Bonovas

Michael A Bookman

Signe Borgquist

William G Bornmann

Francesca Borrelli

Cristina Bosetti

FT Bosman

Frederic Bost

Donald Bottaro

Denise Boudreau

Nancy Boudreau

Judith VMG Bovee

JE Bower

Daniel W Bowles

Carol Box

Karen Brautigam

Boudewijn JM Braakhuis

Loretta Brabin

Thomas Brabletz

Sergio Bracarda

Marc Bracke

Chiara Braconi

Penelope A Bradbury

Kate E Brain

Tommaso Brancato

Jeannine Brant

Donald P Braun

Michael Braun

Thomas Brenn

Hermann Brenner

Walburgis Brenner

Kai Breuhahn

Ryan Brid

John Bridgewater

Louise A Brinton

Carolyn D Britten

Gero Brockhoff

Bertha Brodin

Philip J Brooks

Ewan Brown

Gina Brown

Robert Brown

Adam Brufsky

Laurence Brugieres

Ulrike Bruning

Heather Bryant

Lukas Bubendorf

Markus W Buchler

Krzysztof Bujko

Christopher M Bunce

Franco M Buonaguro

Maximilian Burger

Matthew Burnell

KL Burnstein

Howard A Burris

Barbara Burtness
Phyllis Butow

Graham Byrnes

Gregory Cairncross

Bruno Calabretta

Gloria M Calaf

Michael Caligiuri

Russell C Callaghan

Margaret K Callahan

Matthew Callister

Emiliano Calvo

Alfonso Calvo Gonzalez

David Cameron

Daniel J Canter

Ettore Capoluongo

Giorgio Capuano

Michele Caraglia

Michele Carbone

Chris R Cardwell

Robert W Carlson

Ruaidhri Carmody

Amancio Carnero

Laura Carrassa

Brett S Carver

J Ignacio Casal

Oriol Casanovas

Peter H Cashin

Carlos A Casiano

Philippe A Cassier

Roberto Castelli

Carlo Castoro

JP Cata

Vincenzo Catalano

Helene Cave

Judith Cave

Yvon E Cayre

Jonathan Cebon

Daniela Cecconi

Luca Cegolon

Andrea Cercek

Stefano Cereda

Richard A Cerione

G Cesaroni

GO Ceyhan

Benoit Chabot

Georgina Chadwick

Max Chaffanet

Charles E Chalfant

Anastasia Chalkidou

Anthony J Chalmers

Marc Chamberlain

Catrina Chambers

Victoria Champion

Andrew T Chan

Andrew M Chan

Karen Kar Loen Chan

Michael WY Chan

Stephen Chan

David Chang

Ellen Chang

Jeffrey S Chang

Su-Hsin Chang

Stephen J Chanock

Thomas Charters

Etienne Chatelut

Santosh K Chaturvedi

Ian Chau

Subhash C Chauhan

Ding Chen

Jian-Guo Chen

Jie Chen

Kexin Chen

Pau-Chung Chen

Qing Chen

Tian-Jian Chen
Wendy Y Chen

$\mathrm{X}$ Chen

Yi-Ting Chen

Yuming Chen

Zhenguang Chen

Simon Cheng

TY Cheng

Ya-Wen Cheng

Louis Chesler

John Chester

Tony Cheung

Maria Dolores Chiara

Tsutomu Chiba

Frank Chinegwundoh

Junzo P Chino

Olivier Chinot

Susanna Chiocca

Gabriela Chiosis

Shih-Hwa Chiou

Julia Clare Chisholm

Satish Chitneni

Rowan T Chlebowski

Byoung Chul Cho

Eunyoung Cho

$S$ Chockalingam

Doo Ho Choi

Ji-Yeob Choi

Toni K Choueiri

Peer Christiansen

SE Christmas

Gerhard Christofori

Shu-Chun Chuang

Jessica Chubak

Leland Chung

Fortunato Ciardiello

YB Cihan

Flora Cimmino

Luca Cindolo

Saverio Cinieri

Andrew R Clamp

Jonathon Clark

Gary Clark

Peter E Clark

Robert Clarke

Christine Clavel

Hope Clayton

James M Cleary

Nicholas J Clemons

Tess Clendenen

William A Cliby

Sally Clive

Philip J Coates

Pierluigi Cocco

Robert J Coffey

Roger B Cohen

Joshua A Cohn

Begona Colas

Graham Colditz

Andrew J Coldman

LA Cole

John Colerangle

Helen M Coley

Marco Colleoni

Danny Colombara

Amedeo Columbano

John T Comerci

John Condon

James L Connolly

Michael B Cook

Colin S Cooper

Mhairi Copland

Domenico Coppola

Pippa G Corrie

Max Costa

Eithne Costello

Bettina Couderc

Andrew Covele

Brendon J Coventry

Jim Coward

Fiona Cowie

Simon J Crabb

Howard C Crawford

Ian A Cree

Roland S Croner

Deirdre P Cronin-Fenton

Emma Crosbie

Neil A Cross

Christina-Maria Cruciat

Andrew BC Crumley

Zoran Culig

Daniel J Culkin

BS Cummings

David Cunningham

Ed Curry

Karen Curtin

Nicola Jane Curtin

Jack Cuzick

Jan Dabritz

Richard D'Andrea

Michael D'Angelica

Antonio B D'Assoro

Maurizio D'Incalci

Charlotta Dabrosin

Rajvir Dahiya

Bjorn Dahlback

Bingbing Da

Maria Grazia Daidone

Angus G Dalgleish

Chendil Damodaran

Janet E Dancey

Sarah Danson

Sarah Darby

Kathleen M Darcy

Joseph Davar

Eduardo Davila

Shaheenah Dawood

Lesley Dawson

Ashley Day

D Day

Pradip De

Geertruida H de Bock

Johann S de Bono

Vincenzo De Feo

Rianne de Gelder

Ugo De Giorgi

Antonio Garcia de Herreros

Frank de Jong

Steven de Jong

Annelies de Klein

Harry J de Koning

Michel de Lorgeril

Ruggero De Maria

Ramon Andrade Bezerra de Mello

Claire de Oliveira

Michele De Palma

Theo M de Reijke

Hugues de The

Nunziatina De Tommas

Elisabeth GE de Vries

Olivier De Wever

Niek J de Wit

Ronald de Wit 
Abdullah Demirtas

Jean-Baptiste Demoulin

Xingming Deng

David T Denhardt

Carsten Denkert

Paul Dent

$S$ Derchain

Subhojit Dey

John Di Guglielmo

Giuseppe Di Lorenzo

Marina Pasca di Magliano

Massimo Di Maio

Francesco Di Virgilio

Eleftherios P Diamandis

Jennifer R Diamond

Luis A Diaz Jnr

Analisa DiFeo

Frederic DiFiore

Eveline M Dijkgraaf

Kimberley Dilley

Farhat VN Din

Charles Dinarello

Daniela M Dinulescu

Caroline Diorio

Luc Yves Dirix

Piet Dirix

Andrea Discacciati

Cholpon Djuzenova

Katalin Dobra

Rosario Donato

Haidong Dong

Conan Brian Donnelly

DW Donnelly

Jose Dopeso

Wolfgang Doppler

Valerian Dormoy

Isabel dos Santos Silva

Julian Downward

Mitch Dowsett

Hisashi Doyama

Mary-Anne Doyle

Gerald Draper

Quansheng Du

Yuri Dubrova

Jozsef Dudas

Andrew C Dudley

Stefan Duensing

Florence Duffaud

Michael J Duffy

Stephen W Duffy

Jonathan S Duke-Cohan

Leonidas H Duntas

SB Dusetzina

Janice P Dutcher

Fraser Duthie

Uma Duvvuri

Helena Earl

Martin M Eatock

Diako Ebrahimi Mohammadi

Andre Eckardt

Kristin A Eckert

Angela B Edgar

Dylan R Edwards

Joanne Edwards

Simone Eerenstein

Suzanne Egyhazi Brage

Hans-Georg Eichler

Heather Eliassen

Sam Eljamel

RE Ellsworth

Jon D Emery

Cathy Eng

Christoph Engel

Ellen G Engelhardt
Hideki Enokida

Alan L Epstein

Suleyman Ergun

Mikael Eriksson

Ferry ALM Eskens

Marie-Louise Essink-Bot

Francisco J Esteva

DGR Evans

William K Evans

Marianne Ewertz

Shereen Ezzat

Susanne Fussel

Luca Fabris

Carole Fakhry

Claire Falandry

Stephen J Falk

Junbao Fan

Shona C Fang

Jesika S Faridi

Farhood Farjah

Angelica Fasolo

Matteo Fassan

Jerome Fayette

Veronika Fedirko

Barry W Feig

Jaime Feliu

Emanuela Felley-Bosco

Annika Fendler

Gong Feng

Cristiano Ferlini

Manuela Ferracin

Gabriella Ferrandina

Daris Ferrari

Robert L Ferris

Maria Feychting

Valentina Fiano

Juan J Fibla

John K Field

William Figg

AI Filiz

Sirio Fiorino

Matthew A Firpo

Jens Fischer

PB Fisher

Harold Fisk

Florian Fitzal

Keith T Flaherty

Gini Florence Fleming

Ian N Fleming

Olivia Fletcher

Raja M Flores

Kirsty Flower

Emmanouil Fokas

Constantino Fondevila

Hugo Ford

Tim Forshew

Martin Forster

Orazio Fortunato

Theodoros Foukakis

William D Foulkes

George Fountzilas

Laure Fournier

Marialuisa Framarino dei Malatesta

Brigitte Franc

Silvia Franceschi

Giulio Francia

Jan Franko

Wolf Herve Fridman

Terence Friedlander

Patrick Frost

JP Fruehauf

Haian Fu

Jie Fu

S Fuji
Mayumi Fujita

Naoya Fujita

Hitoshi Fujiwara

Masashi Fukayama

Peter Fuller

Martin Gotte

Christina Gade

Peter Gahan

Nicolas Galazis

Carlotta Galeone

Domenico Galetta

Ferdia Gallagher

Gary E Gallick

Eve Gallop-Evans

Luca Galluzzi

Antoine Galmiche

Daniel A Galvao

Laura Gamble

Ram N Ganapathi

Giorgio Gandaglia

Patricia Ganz

Sizhi Paul Gao

YanJing Gao

Ying Gao

Yuzhen Gao

Claus Garbe

Julio Garcia-Aguilar

Montserrat Garcia-Closas

Angel M Garcia-Lora

Cedric F Garland

Christopher R Garrett

Christoph Gasche

K Gately

Gemma Gatta

Pascal Gauduchon

Pathria Gaurav

Paul R Gavine

Andrew Gaya

Mark Gaze

Maria Gazouli

Susan Gearhart

Olaf Gefeller

Ju Ian Geh

Hans Gelderblom

Fernand-Pierre Gendron

Aleksandra Gentry-Maharaj

Christopher Gerner

Stanton L Gerson

Andreas J Gescher

David A Gewirtz

Michael Ghadimi

Sadaf Ghaem-Maghami

Amr Ghaleb

Paula Ghaneh

Francois Ghiringhelli

Paramita M Ghosh

Georgios Giamas

Bruce Giantonio

Faith Gibson

Spencer B Gibson

Michael Gierth

Ziv Gil

Duncan Gilbert

Michael Z Gilcrease

Jonathan Gill

David Gillespie

Antonio Giordano

Sharon H Giordano

Elisa Giovannetti

Edward L Giovannucci

Maria Dolores Giraldez

Geoffrey Girnun

Rosalind Margaret Glasspool

Robert I Glazer

Jonathan M Gleadle

Olivier Glehen

Hilary Glen

Paul Glen

Bengt Glimelius

Stefan Gluck

Rob Glynne-Jones

WT Godbey

Sanjay Goel

Talia Golan

Laura S Gold

Alfred L Goldberg

L Goldin

Daniel Goldstein

Marc Gollub

Asieh Golozar

Mehra Golshan

Maria Cristina Cintra Gomes-Marcondes

Jaime Gomez-Millan

AK Goncalves

Priscilla Goncalves

Tatsuo Gondo

Zhihong Gong

Celine Gongora

Andrew Gonzalez

Cristina Gonzalez

Ana Maria Gonzalez-Angulo

John L Gore

Steven D Gore

Yves Gorin

Margot A Gosney

James Gossage

Stephen Gottschalk

Ioannis Gounaris

Charles Gourley

Heike I Grabsch

Marian Grade

Janet Graham

Cristina Grange

William B Grant

Claudia Gravekamp

Shannon Graver

Mel Greaves

Beverly B Green

Joel S Greenberger

William Greenhalf

Julia B Greer

Thomas Grewal

Alastair Greystoke

Arjan W Griffioen

Corinne E Griguer

Peter S Grimison

Elizabeth A Grimm

Sergey Grivennikov

Rainer Grobholz

Joanna Groden

Patti Groome

Derek Grose

Line Groth-Pedersen

Viktor Gruenwald

Michael Grusch

Yajia Gu

Fiorella Guadagni

Henk-Jan Guchelaar

Pascal Guenel

Stephen Guest

Chandan Guha

Neela Guha 
Lin Guo

Qi Guo

Ya-Jun Guo

Avinash Gupta

Digant Gupta

Merih Guray Durak

Britt Gustafsson

Mark D Gustavson

Michael Haas

Rick L Haas

Neville F Hacker

Ulrich Thorsten Hacker

Thorsten Hagemann

Bengt Hallberg

Daniel G Haller

Christian Hallermann

Steve Halligan

Toshihisa Hamada

Marije E Hamaker

Chisato Hamashima

Stanley R Hamilton

William T Hamilton

Peter S Hammerman

Adrienne M Hammill

F Han

Wonshik Han

Aaron Hansen

Torben Frostrup Hansen

Yasuaki Harabuchi

Nadia Harbeck

Janet R Hardy

Pirkko Harkonen

Shelley Harris

Ewen Harrison

Simon J Harrison

Daniel Hartmann

Helen J Hathaway

Christos Hatzis

Ulrike Haug

Stefan Hauser

Karin Haustermans

Maria A Hawkins

Michael Max Hawkins

Shannon M Hawkins

Andrew Hayes

Brian Haylock

Biao $\mathrm{He}$

$\mathrm{Xia} \mathrm{He}$

Susan Heavey

Jaclyn F Hechtman

David W Hedley

Eveline AM Heijnsdijk

Sirpa Heinavaara

Rebecca S Heist

I Hellstrom

Andrew E Hendifar

Daniel Yick Chin Heng

Jan G Hengstler

N Lynn Henry

Dae Seog Heo

Georges Herbein

C Hernandez-Giron

Ana Maria Hernandez

Ingrid Herr

Viviane Hess

Rodney John Hicks

Ernest Hidalgo

Geoff Higgins

Nobuko Hijiya

Mark Hill

Mika Hilvo

Satoshi Hirano

Falk Hlubek

Marko Hocevar
Daniel Hochhauser

Howard S Hochster

Bianca AW Hoeben

Geir Hoff

Erin Hofstatter

Janneke Hogervorst

Stefan Holdenrieder

ME Holick

Sten Holmang

Lars Holmberg

Sarah V Holt

David S Hong

Dave SB Hoon

Kirsten Hopkins

John Hopper

Norman Hord

Adilia Hormigo

Alan Horwich

Peter J Hoskin

Nadine Houede

E Andres Houseman

Gilles Houvenaeghel

Liang-Tsai Hsiao

Joseph Hsu

Dingzhi Huang

H Huang

Jing Huang

Weei-Yuarn Huang

Richard B Hubbard

Stephan Huber

Robert A Huddart

JG Hugtenburg

Richard Hunt

Aliya N Husain

Roberto Iacovelli

Rosy Iara Maciel de Azambuja Ribeiroa

Ash Ibrahim

Daisuke Ichikawa

Shingo Ichimiya

OK Idowu

Ray Kruse Iles

Martin Illemann

David H Ilson

Fumiaki Imamura

Peter F Infante

Claire Infante-Rivard

Federico Innocenti

Masafumi Inomata

Akira Inoue

Masahiro Inoue

PD Inskip

Martino Introna

Hamish Ireland

Viacheslav Iremashvili

Meredith S Irwin

Ken Ishitani

Jorma Isola

Amalia M Issa

Antoine Italiano

Kazuyuki Itoh

Yoshiyuki Itoh

Johanna Ivaska

Lene H Iversen

Motoki Iwasak

Prasad Iyer

Arnaud Jaccard

David Jackson

William Jacot

Ulrich Jaehde

Ishmael A Jaiyesimi

Anders Jakobsen

Mark J Jameson

Nigel B Jamieson

Hay Janas
Michelle C Janelsins

Filip Janku

Wolfgang Janni

Emiel AM Janssen

David F Jarrard

Milind Javle

J Jayasekera

Gordon C Jayson

Youjin Je

Mona Jeffreys

Ernest Jehangir

Hanne Krogh Jensen

Jorgen Bjerggaard Jensen

Hyeon Jeong

Milbum J Jessup

Jun Yuan Ji

Lu Jiachun

Xuejuan Jiang

Yixing Jiang

Bernd Jilma

Antonio Jimeno

Duncan Jodrell

Heikki Joensuu

Leif Johansson

Colin Johnson

Ian Johnson

Lisa G Johnson

Peter WM Johnson

Philip J Johnson

Frank E Jones

George Don Jones

Jennifer Jones

Kevin B Jones

Lee W Jones

Rena Jones

Robert J Jones

Robin L Jones

Marjolijn Jongmans

Bengt Jonsson

Benedicte Jordan

Ekaterina S Jordanova

Manish Joshi

Woong Ju

Lucienne Juillerat-Jeanneret

Eva Jungel

Martin Kobel

Rudolf Kaaks

Johannes H Kaanders

Kyuichi Kadota

Keita Kai

Bernd Kaina

Matthew F Kalady

Miklos Kalapos

Marinos Kallikourdis

Raghu Kalluri

Gabriela Kalna

Takao Kamei

P Kameritsch

Akihide Kamiya

Nobuhiro Kanaji

Chryso Kanthou

Sujala Kapur

Christos S Karapetis

Thomas Karn

Michael Karsy

Anna Kashina

Efstathios Kastritis

Goulnar Kasymjanova

Hiroaki Kataoka

Kikuya Kato

Michael W Kattan

Aaron E Katz

Scott H Kaufmann

Ilias G Kavouras

Masaaki Kawahara

Kazuyuki Kawakami

Paul Keeley

Sean Kehoe

Michael Keidar

Muireann Kelleher

Charles Keller

Kevin M Kelly

Karl T Kelsey

John Kemshead

Kristen Kennedy

Richard Kennedy

Paraic A Kenny

Ruth A Keri

David Kerr

Timothy J Key

Carolina Khalid-de Bakker

Iftekhar Khan

Seema A Khan

Leeka Kheifets

Bernard Khoo

Eiji Kikuchi

Luciana Kikuchi

Yoshihiro Kikuchi

Christopher Kim

Jung Chul Kim

Sun Young Kim

Young-Sik Kim

Leo J Kinlen

Akiyoshi Kinoshita

Yoshikazu Kinoshita

Benjamin R Kipp

Yasuhiko Kitadai

Muaiad Kittaneh

Jorg Kleeff

P Klepstad

Carolyn Klinge

Thomas Klonisch

Matthis Kloor

Ann H Klopp

Heinz-Josef Klumpen

Jonathan PS Knisely

Emily Ko

Lena Koch

Hemant M Kocher

Leo Koenderman

Yoshikatsu Koga

Donat Kogel

Holbrook E Kohrt

E Kokkotou

Jill Kolesar

Masaaki Komatsu

Shuhei Komatsu

Raymond L Konger

Hiroyuki Konishi

$\mathrm{H}$ Koon

Bridget Koontz

Jurgen Kopitz

Murray Korc

Antonis E Koromilas

Joanne Kotsopoulos

Michael I Koukourakis

Alexander Kraemer

Onno Kranenburg

Bernd J Krause

Tibor Krenacs

Nils Kroeger

Mary E Kroll 
Kaoru Kubota

Alex Kudrin

Krystle Kuhs

George Kulik

Athan Kuliopulos

Addanki P Kumar

Rajiv Kumar

Shaji Kumar

Ujendra Kumar

Chandan Kumar-Sinha

Gopal C Kundu

Chikara Kunisaki

Han-Pin Kuo

Hann-Chorng Kuo

Michael E Kupferman

Peter Kuppen

Manabu Kurokawa

Jean-Emmanuel Kurtz

Dina M Kweekel

Pingling Kwok

Natasha Kyprianou

Maria Kyrgiou

Carlo La Vecchia

James V Lacey, Jr

Barry J Laird

KC Lakshmaiah

Paul C Lambert

Matteo Lambertini

Diether Lambrechts

Pietro Lampertico

Olivier Lamy

Johnathan M Lancaster

Stephanie R Land

Charles N Landen, Jr

Loic Lang-Lazdunski

Simon P Langdon

Ruth E Langley

Cord Langner

B Lankat-Buttgereit

James M Larkin

Timothy L Lash

Silke Lassman

Claus Lattrich

Yun-Fai Chris Lau

Joel Laufer

Louise Laurent

Dominique Laurier

Cinzia Lavarino

Matthew W Lawless

Gill M Lawrence

Christophe Le Tourneau

Annette Lebeau

Andre Lechel

Fabrice Lecuru

Jonathan A Ledermann

Anne WM Lee

Ching-Chih Lee

Jae Cheol Lee

JM Lee

Te-Chang Lee

Ulrich Lehmann

Yoav Leiser

Marcis Leja

Jochen K Lennerz

ME Leon

Herbert Lepor

Antoon (Tony) Edouard Lerut

Gregory B Lesinski

Giberto Ka Kit Leung

Ricky Leung

Fabio Levi

Stephen Lewis

Joan Lewis-Wambi

Catherine Lhomme
Jingmei Li

Min Li

Wen-Qing Li

Ye-Xiong Li

$\mathrm{Yi} \mathrm{Li}$

Yong $\mathrm{Li}$

Zhe-Xiong Lian

Dan Liebermann

GJ Liefers

Torsten Liersch

James W Lillard, Jr

Carmen Silva Passos Lima

Jin-Ching Lin

Ming-Chia Lin

Tao Lin

Zongming Lin

Annika Lindblom

Katja Lindel

Colin Lindsay

Linda Sofie Lindstrom

Hui Ling

Sabine K Linn

Michael Linnebacher

Eleni Linos

Steven M Lipkin

W Ian Lipkin

Marc Estes Lippman

Bolin Liu

Chien-Ying Liu

Fei-Fei Liu

Glenn Liu

$\mathrm{Hu}$ Liu

Lianxin Liu

Meng-Zhong Liu

Qingyun Liu

Rui Liu

Stanley K Liu

Xiangguo Liu

Xuedong Liu

Ingrid Lizarraga

Gustaf Ljungman

Xavier Llor

Hui-Wen Lo

Jeng-Fan Lo

Siu Hing Lo

Sharon Lobert

Fiona Lofts

Mignon Loh

Sherene Loi

Anna E Lokshin

Thomas London

Wendy B London

Adhemar Longatto-Filho

Herbert Loong

Antonio Lopez-Beltran

Luis A Lopez-Fernandez

Esther Lopez-Garcia

Armando Lopez-Guillermo

Fernando Lopez-Rios

Florian Lordick

Yohann Loriot

Joannie Lortet-Tieulent

Pat Lorusso

Emil Lou

Katherine Loveland

Philip S Low

Hua Lu

$\mathrm{K} \mathrm{Lu}$

Shan Lu

Zuxun $\mathrm{Lu}$

Jay Lubin

Sam J Lubner

Chad Luedtke

Alessandro Lugli
Marta Lukaszewicz-Zajac

Philip Lupo

Brigid Mary Lynch

Craig Lynch

HT Lynch

Elsebeth Lynge

Georgios Lyratzopoulos

Athena Matakidou

Daniela Matei

John Mathews

Ron HJ Mathijssen

Xavier Matias-Guiu

Keitaro Matsuo

Koji Matsuo

Katherine Matthay

Marie-Helene Mayrand

Tapati Mazumdar

Nathalie Mazure

Massimiliano Mazzone

Peter J Mazzone

Martin McCabe

John L McCall

Elaine McCartney

AL McCleary-Wheeler

Hayley McDaid

Duncan McLaren

Catriona McLean

Anne McLeer

Martin McMahon

Andrew McMillan

Donald C McMillan

Keely May McNamara

Mairead McNamara

Iain A McNeish

Sean McPhail

Robert McWilliams

Kapil Mehta

Alfons Meindl

Peter Meldgaard

Bohuslav Melichar

Albert Mellick

Stephen Meltzer

Christine Menetrier-Caux

Lourdes Mengual

Kavitha Menon

E Merler

Melissa A Merritt

Wilma E Mesker

Leonid Metelitsa

Tim Meyer

Paul A Meyers

Michael Michael

Martin Michaelis

Karin B Michels

Carine Michiels

Stefan Michiels

Patrick Micke

Julie Midtgaard

Michele Milella

Anne Miles

David W Miles

A Miller

Joshua Miller

Ian G Mills

Michael Millward

Roger Milne

Do Sik Min

Toshinari Minamoto

John Minna

Giuseppe Minniti

Bruce D Minsky

Alexander Mirnezami

Harald Mischak

Sandip K Mishra

Shiraz I Mishra

Benjamin Misselwitz

James B Mitchell

John S Mitcheson

Koji Miyahara

David Miyamoto

Masaru Miyazaki

Holger Moch

Helmout Modjtahedi

Beatrice Mohelnikova-Duchonova

Tony SK Mok

Alex Molasiotis

Damian J Mole

L Rhoda Molife

Michele Molinari

Florence Molinie

Henrik Moller

Timothy Molloy

Maurizio Mongiat

Pierre Mongiat-Artus

Bradley J Monk

Filippo Montemurro

Guy H Montgomery

R Bruce Montgomery

Caroline Moore

Richard G Moore

Anna Moretti

Carys Morgan

Richard Morgan

Philippe Morice

Masaru Morita

David Morris

Jo Morrison

Muriel Moser

Marsha A Moses

Chaya Moskowitz

Sue M Moss

Susan J Moug

Sujana Movva

Barbara Mroczko

Mustafa-Zelal Muallem

Lorelei A Mucci

Susette Muellers

Franco Muggia

Colin R Muirhead

Priyabrata Mukherjee

Hasan Mukhtar

Thomas Muley

Paul B Mullan

Alastair John Munro

Peter Murchie

Jane Muret

Caitlin C Murphy

Graeme Murray

Lara Mussolin

Anna Maria Musti

JS Myers

Ronald E Myers

Ghulam Nabi

Takahide Nagase

Gabriele Nagel

Christina Nagle

Seema Nagpal

Mitsutoshi Nakada

Masayuki Nakagawa

Apostolos Nakas

Joo-Hyun Nam

Ghoutham Narla 
Peter S Nelson

Dario Neri

Kirsten Ness

Gila Neta

Efrat Neter

Manfred Neuberger

Tom Newsom-Davis

Robert U Newton

Wai-Tong Ng

Paddy Niblock

Hazel B Nichols

Steve Nicholson

Hans Jorgen Nielsen

M Angela Nieto

Daniel Nilsson

Yasuhiko Nishioka

Tomoyuki Nishizaki

Donato Nitti

Andrew B Nixon

Vincent CO Njar

Mette Norgaard

Nicola Normanno

Remi A Nout

Silvia Novello

Masahiro Nozawa

James O’Beirne

Mary Ann O'Brien

Joseph O'Flaherty

Mairin O'Mahony

Ruth M O'Regan

Eileen Mary O'Reilly

Michael O'Rorke

Sandra O'Toole

Andreas Obermair

Christoph Oesterreicher

Birgitte V Offersen

Shuji Ogino

Takashi Ohtsuka

Karin Oien

Veronica Ojetti

Hirohisa Okabe

Alicia Frances Clare Okines

Alexander Olawaiye

Steven Edward Oliver

Ivo A Olivotto

Tracy Onega

Adedayo Onitilo

Akira Ono

Fabio Orlandi

Steffen Ormannns

Torben F Orntoft

Nicholas Orr

Richard Osborne

Clodia Osipo

Megan Othus

Eigo Otsuji

Christian Ottensmeier

Michael J Overman

Geoffrey R Oxnard

Mototsugu Oya

Michio Ozeki

Simon Pacey

Pal Pacher

Eugenio Paci

Karen Page

Rish K Pai

Sumanta Kumar Pal

Claudia Palena

Richard Palmqvist

Jianji Pan

Demosthenes B Panagiotakos

Maria Panchenko

Hardev S Pandha

Deepika Pandhi
Tianxiang Pang

Klaus Pantel

Nickolas Papadopoulos

Olivier Pardo

Joana Paredes

Neehar Parikh

Jun Yong Park

Song-Yi Park

Eric Kenneth Parkinson

Christine L Parr

Toshima Z Parris

Boris Pasche

Nora Pashayan

Eddy Pasquier

Harvey I Pass

JR Passweg

Marcal Pastor-Anglada

Ugo Pastorino

Gaurav Pathria

Prashant B Patil

Ingvild Paur

John M Pawelek

Yudi Pawitan

Jennifer Payne

Mick Peake

Adali Pecci

Marc Peeters

Gang Pei

H Peinado

Frank Peinemann

Claudio Pelucchi

Luiz O Penalva

Nicolas Penel

Hanxiang Peng

David F Penson

George Pentheroudakis

E Penuel

Silvia Penuela

Massimo Perachino

PC Pereira

Stephen P Pereira

Edith A Perez

Marianela Perez

R Allen Perkins

Stephanie M Perkins

Francesco Perrone

Godefridus J Peters

Russell D Petty

Ludovic Peyre

Francesco Pezzella

Christopher M Pezzi

Lawrence M Pfeffer

Ulrich Pfeffer

Ruth M Pfeiffer

Paul D Pharoah

Marx-Stoelting Philip

Philip A Philip

Kelly-Anne Phillips

Roger M Phillips

Amanda I Phipps

Didier Picard

Piero Picci

Martin Pichler

Joel Picus

Jean-Yves Pierga

Marco Pierotti

Kristian Pietras

Jean-Pierre Pignon

Linda M Pilarski

Dietmar Pils

Nicola Pimpinelli

Pascal Pineau

Robert Pirker

Tobias Pischon
Vito Pistoia

Fiona Pixley

Marie Plante

Ludwig Plasswilm

Ruben R Plentz

Andrzej Plewka

Chris Plummer

Igor P Pogribny

Venerino Poletti

Jessica Poon

Sanjay Popat

E Poplin

Camillo Porta

Edwin Posadas

Isabel Poschke

Marshall Posner

Michael Postow

Vanessa A Potter

Julio Pow-Sang

Arfon GMT Powell

Aleix Prat

Timothy J Price

John N Primrose

J Howard Pringle

Catrin Pritchard

Kathleen I Pritchard

Kathy Pritchard-Jones

Giuseppe Procopio

David Propper

Jennifer Prosperi

Amanda Psyrri

Eero Pukkala

Dianne Pulte

Mark P Purdue

Raj Puri

Samantha L Quaife

Denis Querleu

Morten Quist

J Ruschoff

Saskia Rademakers

James M Rae

Sajjad Rafiq

Nuh Rahbari

Gazanfar Rahmathulla

Venu Raman

Anand K Ramasubramanian

Cody Ramin

Ajay Rana

Vivek Rangnekar

Juha Rantala

Sheela Rao

Annamaria Rapisarda

Armin Rashidi

Eric Raymond

DJ Raz

Shahzad Raza

Albiruni RA Razak

FX Real

Vinciane Rebours

Rishindra Reddy

Christopher PF Redfern

Gregory A Reed

Nicholas Simon Reed

Simon Reed

Helen Reeves

M Reiss

Andrew G Renehan

Ramesh Rengan

Matilda Rentoft

Miriam Reuschenbach

Gary W Reuther

SL Revels

Brent Rexer

Marc A Reymond

Andrew R Reynolds

Luigi Ricciardiello

MA Richard

Jennifer K Richer

Mothaffar F Rimawi

Brian I Rini

Michael Rink

HA Risch

Janet M Risk

Licia Rivoltini

K Thomas Robbins

Benjamin M Robinson

Martin Robinson

Max Robinson

Carissa Rocheleau

Andrew W Roddam

Claire Rodriguez-Lafrasse

Lisa R Rogers

Charlotte Rolny

Joan M Romano

Margarita Romeo

Guglielmo Ronco

Monique Roobol

Martin Roosli

Patrik Rorsman

Derek J Rosario

Peter W Rose

Rafael Rosell

Eliot M Rosen

Sara E Rosenquist

Brian D Ross

James A Ros

Alonzo H Rossi

Giulio Rossi

Marta Rossi

Christophe Rosty

Arnaud D Roth

Brian G Rowan

Campbell SD Roxburgh

Patricia Roxburgh

Greg Rubin

Kathryn J Ruddy

Charles M Rudin

M Ruhul Quddus

Simon Rule

Valerie W Rusch

Lesley Rushton

Kjell Magne Russnes

Antonio Russo

Gian Luigi Russo

Vincenzo C Russo

Emiel J Rutgers

Piotr Rutkowski

Sascha Rutz

Steve Ryder

Byungwoo Ryu

Sepideh Saadatmand

Anna Sablina

Paola Sacerdote

M Sachdeva

Adrian Sacher

Michael Sachs

Satoru Sagae

Erik Sahai

Nagahiro Saijo

Rajiv Saini

Kathleen M Sakamoto

Joseph K Salama 
Helene Sancho-Garnier

Dario Sangiolo

Graciela Santillan

Alessandro D Santin

Daniele Santini

Victoria Sanz-Moreno

Anna Sapino

Diana Sarfati

Daniel J Sargent

Fazlul H Sarkar

Sibaji Sarkar

Debashis Sarker

Hiroki Sasaki

Peter Sasien

Yasufumi Sato

Taroh Satoh

Mark P Saunders

Nicolai E Savaskan

Elinor J Sawyer

Giorgio Scagliotti

Aldo Scarpa

Mario Scartozzi

Kurt Schalper

Werner Scheithauer

Laura Scherer

Alfred Schinkel

Johannes A Schmid

Tobias Schmid

Manuela Schmidinger

Hans-Joachim Schmoll

A Robert Schnatter

Robert J Schneider

Heiner Schrewe

Christoph Schuhmacher

Wolfgang A Schulz

Udo Schumacher

Joachim Schuz

Anthony Schwarer

Lukas Schwingshackl

Francesco Sclafani

Carolyn Scott

Kieran Scott

Christopher Scrase

Nadja Seidel

Naohiko Seki

Gregg L Semenza

Stephen Senn

Peter Senter

Karen Sepucha

Natalie Serkova

Diego Serraino

Cristiana Sessa

Ivana Sestak

Gautam Sethi

V Wendy Setiawan

Thomas Seufferlein

Karen Sfanos

Dennis C Sgroi

Alaaeldin Shablak

Manish A Shah

Mahvash Shahidi

Masood Shammas

Smruta Shanbhag

Jenny Shannon

Chun-kui Shao

Chunlin Shao

Rong Shao

Dipali Sharma

Geetanjali Sharma

Ricky A Sharma

Rohini Sharma

Richard Sharpe

Jacqueline A Shaw

Richard John Shaw
Maw-Chang Sheen

Guo-Ping Shen

Lin Shen

Linda A Sherman

Chanjuan Shi

Qian Shi

Xuebing Shi

Tatsuhiro Shibata

Anthony F Shields

Katsumi Shigemura

Xianglin Shih

Hideaki Shimada

Muneaki Shimada

Janet Shipley

Susan Short

Rachna Shroff

Xiao-Ou Shu

Nicola Sibson

Amen Sibtain

Florian Siebzehnrubl

Abby B Siegel

Markus David Siegelin

Cornelis FM Sier

Ana Elizabete Silva

Andrew Silver

Alice E Simon

Julian Simon

Michael Simon

Melanie Simpson

Tomas Simunek

Ajay Pratap Singh

Harmeet Singh

Preet Paul Singh

Siddharth Singh

Tristan Sissung

Lillian L Siu

Tina Louise Skinner

Finn Egil Skjeldestad

Rolf I Skotheim

Ondrej Slaby

Mark Slade

Jonathan Sleeman

Stefan Sleijfer

Erica Sloan

Keiran Smalley

Karel Smetana

Alan Smith

Christopher Smith

Laura Smith

Megan Smith

Robert Smith

Sophia K Smith

John Washburn Smith II

K Smith-McCune

Elizabeth C Smyth

Silvia Soddu

Riccardo Soffietti

CT Sofocleous

Abhishek A Solanki

Eduardo Solsona

Neeta Somaiah

Samir Sonej

Guru Sonpavde

Richie Soong

Prasanna Sooriakumaran

Bengt Sorbe

Kjetil Soreide

Claus Sorensen

Melissa Southey

Teresa Spadea

Giulio Spagnoli

Paul N Span

Alex Sparreboom

James F Spicer
Johnathan Spicer

Sarah Spiegel

Diana Spiegelberg

Ben Spycher

Jeremy A Squire

Nadadur S Srikanth

Sanjay K Srivastava

Brad St Croix

Karin Stalberg

Maciej Stepnik

Brendan C Stack

John Staffurth

Michael Stahl

Meir Stampfer

Jeffrey Stanley

Giorgio Stanta

GR Stark

Justin Stebbing

Neeltje Steeghs

Colin W Steele

Nicola Steele

Robert JC Steele

VR Steger

Alexander H Stegh

Mark N Stein

Konstantinos Stellos

Marta Stelmach-Mardas

Sally Stenning

Kristina Stenstedt

Alex Stepan

Mariana Stern

William G Stetler-Stevenson

Richard G Stevens

Clare Stevinson

Alexandra Stewart

Grant Stewart

Simon Stewart

Ewout W Steyerberg

Sebastian Stintzing

Jon Stobo

Gabriele Stocco

Carol Stocking

Robert Stoehr

Rachael Stolzenberg-Solomon

Dawn Storey

Michael Stotz

Dirk Strauss

William Ben Strean

Sofie Struyf

Martijn Matthias Stuiver

Roger Stupp

Vivek Subbiah

Dharmalingam Subramaniam

Subbaya Subramanian

Stefan Suciu

Amit Sud

Tamotsu Sudo

Paul H Sugarbaker

Toru Sugiyama

Paniti Sukumvanich

Helen W Sullivan

Ryan Sullivan

H Sultmann

Vera Suman

Katherine Sumpter

Qingmin Sun

Malin Sund

Karin Sundstrom

Fung-Chang Sung

Hiromu Suzuki

Mattias Svensson

Charles Swanton

John Sweetenham

Anthony J Swerdlow

Daniel Swinson

Wing-Kin Syn

Tibor Szarvas

Peter Szlosarek

Mario Sznol

Ewa Szumacher

Tsutomu Tabata

Ayumu Taguchi

Emanuela Taioli

Kazuaki Takabe

Masashi Takano

Seiichi Takenoshita

Yuichi Takiguchi

Iannis Talianidis

Rulla M Tamimi

Martin Tammemagi

Su-Ming Tan

XL Tan

Shinji Tanaka

Damu Tang

Yungan Tao

Alessandra Tavani

Andrei Tchirkov

Carmen S Tellez

Alexei Tepikin

K Teramoto

Mathewos Tessema

Fiona Thistlethwaite

Audrey Thomas

David B Thomas

Jeremy St J Thomas

L Thommesen

Patricia A Thompson

Paul Thompson

Mangesh Thorat

Aaron P Thrift

Martin Thurnher

Raoul Tibes

Andre Tichelli

Jim Tiernan

Kaja Tikk

Madeleine MA Tilanus-Linthorst

Vesna Todorovic

Philip J Tofilon

Junya Toguchida

Yasushi Toh

Yoshihiko Tomita

Ian PM Tomlinson

Giuseppe Tonini

Alex Tonks

Emily S Tonorezos

Dott Francesco Torino

Garth W Tormoen

David Tougeron

Zahi Touma

Paul Townsend

Gill M Tozer

Philip C Trackman

Toby N Trahair

Cameron C Trenor

Dimitrios Trichopoulos

Manisha Tripathi

KF Trivers

Jakob Troppmair

Jone Trovik

Kiril Trpkov

Donald L Trump

Livio Trusolino 
Richard Calvin Turkington

Claire Turnbull

David Turner

Nicholas Turner

David Tuveson

Chris Twelves

Betty Tyler

Lieve Tytgat

Andrej Udelnow

Frederick R Ueland

Naoto Ueno

Paola Ulivi

Rolland T Ullrich

Giske Ursin

Narikazu Uzawa

Anil Vachani

Jay V Vadgama

Stephan Vagner

Vincenzo Valentini

Nicola Valeri

Daniel Vallbohmer

Laura Valle

NG Vallianou

Anton H Van den Meiracker

A van der Gaast

Bert van der Reijden

Carla $\mathrm{H}$ van Gils

Wim $\mathrm{H}$ van Harten

L Van Kaer

Folkert J Van Kemenade

Flora E van Leeuwen

Katherine Van Loon

Jan van Meerbeeck

Nicolien van Ravesteyn

Leo GM van Rossum

Carter van Waes

Edwin Van Wijngaarden

Nico van Zandwijk

Hugo Vankelecom

Sheelu Varghese

Yogesh K Vashist

Vasilis Vasiliou

Lars J Vatten

E Vazquez

Andrea Vecchione

Peter Vedsted

Kenneth Vega

Guillermo Velasco

Lionne Venderbos

$\mathrm{K}$ Venetsanou

Conchita Vens

Inder M Verma

Sally Vernon

Claire F Verschraegen

Emily Vertosick

Renata Veselska

Vanessa Medina Villaamil

Paolo Vineis
Rosa Visone

Francisco J Vizoso

Virginie Vlaeminck-Guillem

Antonia Vlahou

Christian von Wagner

Alina Vrieling

Ivana Vucenik

Danielle J Vugts

Tom Waddell

David Walker

James A Walker

Marjorie Walker

Lucy Wall

Fiona Walter

Stefan Walter

Thomas Walter

Gernot Walter

Hans H Wandall

Chiayeng Wang

Debin Wang

Jean YJ Wang

Jung-Der Wang

Kepeng Wang

Li-Hsuan Wang

Miao Wang

Shaomeng Wang

Xin Shelley Wang

Xuewen Wang

Yisong Wang

Zhen-Jie Wang

Jane Wardle

Saman Warnakulasuriya

Erica T Warner

Arne Warth

Harpreet S Wasan

Masayuki Watanabe

Justin Waters

David Watkins

David I Watson

Deborah Watson-Jones

Penelope M Webb

Thomas Webb

Jeffrey Weber

Daniel S Wechsler

Joseph Tien-Seng Wee

Huafeng Wei

Wilko Weichert

Sebastian Weis

Christian Weiss

Dominik Weiss

Lee welty

De-Sheng Weng

Nicolas Wentzensen

Haim Werner

Catharine ML West

Virginie Westeel

J Westermarck

Shannon Westin
Jeremy S Whelan

Katriina L Whitaker

Alexandra J White

Jeff D White

Theresa L Whiteside

Sophie Whyte

Bertram Wiedenmann

John Wiencke

SM Willems

Ann Caroline Williams

Casey Williams

Gareth Haydn Williams

John HH Williams

Michael Williams

Charles Wilson

Jessica Claire Wilson

Kathryn M Wilson

Malcolm Wilson

Thomas Winder

Lori Wirth

J Alfred Witjes

Penella J Woll

Chi Hang Wong

DT Wong

Kwong-Kwok Wong

Sandra L Wong

Yin Ling Woo

Laurence Wood

Andrew Wotherspoon

David Wotton

Hui-Chen Wu

Jinsong Wu

Kaichun $\mathrm{Wu}$

Kejin $\mathrm{Wu}$

Lisa Wu

Chris Wynne

$\mathrm{F}$ Xia

Yun-Fei Xia

Fang-Yun Xie

Keping Xie

Qian Xie

W Xie

Ning Xu

Ruodan Xu

Sohsuke Yamada

Hiroharu Yamashita

Dongyan Yang

Hiafeng Yang

Hua Yang

Muh-Hwa Yang

Wan-Shui Yang

Xiaoyi Yang

Yand Yang

Gregory A Yanik

Song Yao

Timothy A Yap

Wendell Yarborough

Hideo Yasunga
Clayton Yates

Amir Yazdi

Marc Ychou

Bin Ye

Douglas Yee

Jennifer M Yeh

Jessica Yeh

Shyh-An Yeh

Sai Yendamuri

Anthony T Yeung

Min Yi

Hongmei Ying

Cheng-Har Yip

Linwah Yip

Tomoya Yokota

Atsuko Yoneda

Kazuhiro Yoshida

Hideyuki Yoshitomi

Hye Jin You

Chang-Ho Youn

Mamoun Younes

Bridget Young

Robert P Young

Jun $\mathrm{Yu}$

Xue Qin Yu

W Yuan

Xianglin Yuan

Ryan Yuen

Seok Joon Yun

MF Yyuen

Emily C Zabor

Nadia Zaffaroni

Per-Henrik Zahl

John Zalcberg

Dan P Zandberg

Marco Zappa

Dimitrios Zardavas

Jason A Zell

Christophe Zemmour

Jose P Zevallos

Fenghuang Zhan

J Zhang

Yongliang Zhang

Z-J Zhang

Lei Zheng

Yi-Min Zhu

Christoph C Zielinski

Wolfgang Zimmerman

Ralph Zinner

Oliver Zivanovic

Inti Zlobec

Manuel Zorzi

Luisa Zuccolo

Christian Zuppinger

Wilbert zwart

Ellen Zwarthoff 